\title{
CLASS PREDICTION IN TOXICOGENOMICS
}

\author{
Nandini Raghavan and Dhammika Amaratunga
}

Department of Non-Clinical Biostatistics, Johnson and Johnson Pharmaceutical Research and Development, LLC, Raritan, New Jersey, USA

\section{Alex Y. Nie and Michael McMillian}

Mechanistic Toxicology Group, Johnson and Johnson Pharmaceutical Research and Development, LLC, Raritan, New Jersey, USA

The intent of this article is to discuss some of the complexities of toxicogenomics data and the statistical design and analysis issues that arise in the course of conducting a toxicogenomics study. We also describe a procedure for classifying compounds into various hepatotoxicity classes based on gene expression data. The methodology involves first classifying a compound as toxic or nontoxic and subsequently classifying the toxic compounds into the hepatotoxicity classes, based on votes by binary classifiers. The binary classifiers are constructed by using genes selected to best elicit differences between the two classes. We show that the gene selection strategy improves the misclassification error rates and also delivers gene pathways that exhibit biological relevance.

Key Words: Classification; Cross Validation; Gene expression; Gene selection; Hepatotoxicity; Linear discriminant analysis; Microarray; Normalization; Toxicogenomics.

\section{INTRODUCTION}

Toxicogenomics is a new field, spawned by the current cascade of genomic information and related technologies, that strives to establish the genomic role in a toxic response to a drug, toxin, or some other external stimulus (Nuwaysir et al., 1999). Toxicogenomics has generated more than a passing interest in the pharmaceutical industry because many drug candidates that fail during later stages of drug development (most disturbingly, in late phase clinical trials), do so because of unforeseen toxicities. Early preclinical prediction of such toxicities would permit swift elimination of such compounds from the development pipeline and facilitate a better and more focused drug development program.

At present, toxicogenomics relies heavily on information gathered from DNA microarray experiments, experiments in which gene expression information is

Received August 24, 2004; Accepted November 24, 2004

Address correspondence to Nandini Raghavan, Johnson and Johnson Pharmaceutical Research and Development, LLC, 1000 Rt. 202 S, Room G004, Raritan, NJ 08869, USA; Fax: (908) 526-2567; E-mail: nraghava@prdus.jnj.com 
obtained by probing cellular mRNA with small glass slides (DNA microarrays) containing thousands of cloned genes or oligonucleotides. For a general statistical treatment of DNA microarrays, see Amaratunga and Cabrera (2004) and Speed (2003).

The current main focus of this effort is to develop unique gene expression "signatures" (or gene-based biomarkers) for a set of prototypical compounds known to induce a particular toxic response. The intent is to use these signatures to (1) better understand the biology underlying the toxic response and (2) develop screens to test novel compounds for potential toxicity based on gene expression profiling (McMillian et al., 2004a,b). From a statistical perspective, this task can be formulated as a problem of classification, an area that has been well addressed in the multivariate statistics literature and is well reviewed by Hand (1997) and Hastie et al. (2001). However, certain features of microarray data and certain aspects of this situation make it unclear as to whether standard classification and prediction methodologies can be directly applied.

The data from toxicogenomics microarray experiments consist of gene expression levels organized into a "gene expression matrix." The rows of the matrix correspond to genes (predictors), and each column represents a microarray sample (observation) for a specific compound. Data would be available for several compounds, from a variety of toxicity classes. In addition, there could be covariate information corresponding to the microarray samples (e.g., compound, dose, route of administration, mRNA sample collection time, microarray batch production date, and mRNA hybridization date.) and to the genes (e.g., gene ontology information).

\section{Analysis Issues}

Here we discuss various issues that arise with microarray data, which impact the ability to perform standard statistical analysis in the sense that their net effect on the dataset is to violate some standard of the tenets and assumptions of conventional statistical analysis.

A1. The cost and complexity of experiments involving microarray data have the consequence that the number of replicates is typically small. In our study, each compound was administered to three rats, corresponding to three biological replicates per compound.

A2. In addition, there are a very large number of predictors (in this case, genes), and they exceed the number of available samples by orders of magnitude. This finding, compounded with A1, poses quite a challenge for conventional statistical methods.

A3. The statistical properties of the data also complicate the analysis. Genes tend to be codependent in clumps. Both the within-gene distribution and the geneto-gene distribution are highly skewed.

A4. Different arrays could be affected differently by differences in sample preparation, mRNA amplification, labeling efficiencies, scanner settings, and so on. These can result in nonlinear array effects, which need to be corrected to align the array distributions. 
A5. In addition, because of the large size of the study, array production and experiments had to be split into batches. As a result, extraneous factors, such as print batch lots and hybridization dates, were also found to introduce bias and needed to be corrected before proceeding with the classification.

More specific to the application at hand, are the following issues:

A6. Clinically, the toxicity classes are somewhat nebulous, with subtypes possible (e.g., carcinogenicity could be further subclassified as genotoxic carcinogenicity or nongenotoxic carcinogenicity).

A7. Some compounds may exhibit properties of more than one type of hepatotoxicity; hence, the boundary between toxicity classes is not welldelineated.

A8. Some classes were much more heavily populated than others, leading to differential information regarding the classes.

A9. Because each class consists of distinct compounds, each of which may induce the toxicity in a slightly different way, the classes per se may be quite heterogeneous.

A10. A novel test compound may not belong to any of the classes in the original training data.

The net result of all these issues is that class distributions may be quite diffuse and not readily separable, making the classification problem challenging.

\section{Design Issues}

There are also several experimental design issues, some general to bioassay, and some specific to the study, that could impact the analysis. Although various strategies could be used to create a well-designed toxicogenomics study, cost and resource limitations mean that, in reality, compromises must be made. This limits the number of compounds that could be tested, the number of biological replicates per compound, the number of dosage levels at which compounds are tested, the number of time points at which tissue samples are taken, and so forth.

Each hepatotoxicity class is comprised of multiple toxicants, usually prototypical representatives of the class. Careful selection of a core set of reference toxicants is crucial to the success of a toxicogenomics study. Typically, very high dosages of the compounds are administered to ensure that any transcriptional changes that are precursors to the type of hepatotoxicity associated with the compound are observable. The dosages are based on prior experimentation, with some care taken to ensure that the compound-dosage combination for members of each class are reasonably comparable (i.e., that they are reasonably equitoxic. Different routes of administration (ip vs. po) could imply different internal mechanisms and, hence, possibly different manifestations of gene expression changes; whether they, despite this result, lead to the same toxicity remains an intriguing open question.

The time at which mRNA samples are taken is also crucial. Pilot studies indicated that certain types of toxicities, like peroxysomal proliferation and macrophage activation, could be easily identified via 24 -hour gene expression data. Figure 1 illustrates this. The plot, which shows a three-dimensional projection of samples from three hepatotoxicity classes (peroxysomal proliferators, macrophage 


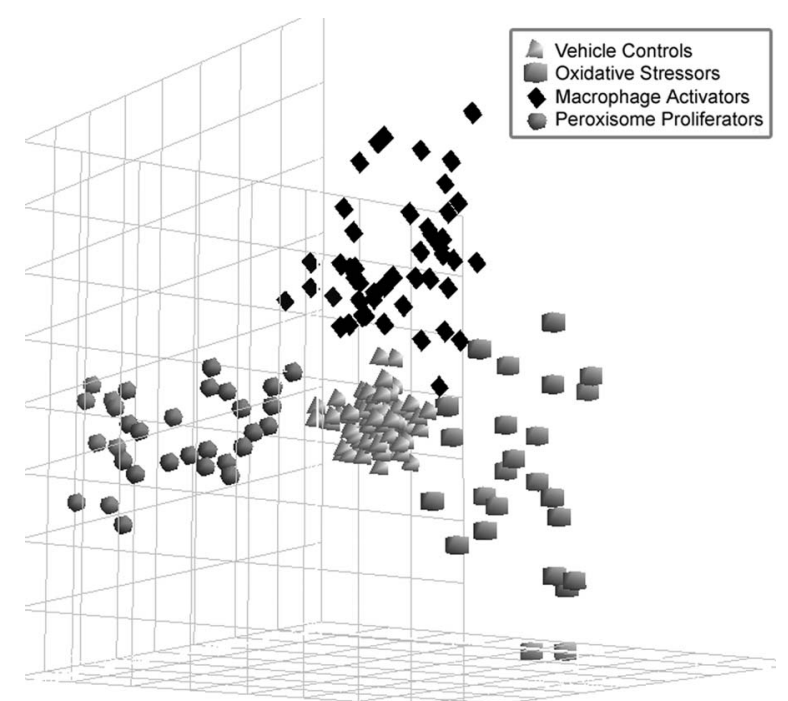

Figure 1 View of samples corresponding to 3 hepatoxicity classes: Macrophage activators, oxidative stressors, and peroxisome proliferators in a 3-D principal components projection.

activators, and oxidative stressors) and vehicle controls in a reduced gene space, indicates that there is clear separation of the classes. However, these classes can also be definitively detected by pathology from short-term toxicity studies. On the other hand, toxicities like carcinogenicity require complex, long-term chronic studies to be detected. One of the issues of great interest is whether such toxicities could also be predicted by using gene expression data collected 24 hours following administration of a very high dose of a test compound. If so, then this procedure could save millions of dollars by screening, and potentially reducing, the number of compounds that need to be studied more rigorously.

\section{Outline of the Analysis Procedure}

The first step in the data analysis is the data preprocessing. This includes identifying and eliminating pathological microarrays; a series of nonlinear normalization steps to align array distributions; winsorizing of outliers; and imputation of missing values. Once these steps are undertaken, the data are ready for analysis and we proceed with the classification procedure.

The classification itself is carried out in two stages. In Stage I, we construct a classifier to classify a compound as toxic or nontoxic. Next, in Stage II, we construct another classifier to classify compounds designated as toxic into the various $(K)$ hepatotoxicity classes. We use a two-step approach to address the $K$-class classification problem in Stage II. In the first step, we perform $K(K-1) / 2$ binary classifications corresponding to each two-class combination of the $K$ classes. In the second step, we collate the votes from the predictions of the $K(K-1) / 2$ binary classifications to make final predictions for each test compound. 
Each of the $(K(K-1) / 2+1)$ binary classifications (note that the classification of compounds as toxic vs. nontoxic in Stage I is also a binary classification problem) comprises two steps:

1. A gene selection strategy to elicit the most relevant genes for differentiating between the two classes (described in greater detail in the section on classification). Given that the number of samples is small and the number of predictors is large, it becomes imperative to do gene selection to reduce the dimension of the covariate space and minimize the possibility of overfitting. Our experiments indicate that the performance of the classifier is significantly improved by prior gene selection.

2. Linear discriminant analysis (LDA) to construct the classifier, after first removing covariate effects from the data. We chose LDA as the classification method because its performance was significantly superior for this problem than other methods such as DLDA [diagonal-LDA, where the covariance matrix is assumed to be diagonal, was recommended by Dudoit et al., 2002]; or LDA following principal components analysis (Amaratunga and Cabrera, 2004); or $k$-NN or other such classifiers. Furthermore, given the tiny replicate-to-predictor ratio, we thought that a linear approach was less likely to overfit the data than a more complex procedure.

The approach of splitting up a $K$-class problem into $K(K-1) / 2$ binary classification problems was proposed by Friedman (1996). He shows that the Bayes rule for the $K$-class problem may be reexpressed in terms of the optimal Bayes rules for the $K(K-1) / 2$ binary classification problems. He suggests that such an approach could lead to substantial gains in accuracy over a $K$-class rule.

In addition, a major advantage of multiple binary classifications vs. a single $K$-class classification for our problem is that gene selection is significantly more effective when we select genes based on pairwise comparisons, rather than a general ANOVA-like comparison among multiple classes. This is particularly true if one of the classes is significantly different from the rest or, if differences between one pair of classes dominates the rest. In such cases, most of the genes selected with an ANOVA approach tend to highlight this particular difference, the low-hanging fruit so to speak, at the cost of more subtle differences.

To validate the procedure, we used leave-one-sample-out and leave-onecompound-out cross-validation. For this, we split the data into training and test datasets. The test dataset was the sample or compound "left out"; the rest functioned as the training dataset. Then we train the classifiers on the training data using the two-step procedure outlined above, make predictions for the test data, and record any misclassifications.

The rest of the article is organized as follows. In the first section, we describe the data. This is followed by a section describing the preprocessing of the data to prepare it for classification. The section on classification and gene selection describes the methodology, including the gene selection strategy, the classification procedure, and the vote-based strategy for final predictions. In the final section, we present the results and discussion for the toxicogenomics dataset that we analyzed. 


\section{DATA DESCRIPTION}

The study that motivated this work involved 61 compounds known to induce hepatotoxicity. They were categorized into eight classes based on the primary type of hepatotoxicity each was known to induce. The objective of the study was to determine their effect on rat gene expression profiles, in particular in rat liver. The selection of the compounds and the assignment of each compound to a toxicity class were based on a careful review of the toxicology literature. In fact, many compounds were prototypes for certain toxicological classes of interest.

Each compound was typically administered to three rats, at a dosage predetermined to be toxic. There were also several vehicle controls run concurrently to establish baseline gene expression levels. The livers were harvested 24 hours following dosing. The mRNA extracted from each liver tissue was amplified and hybridized to four cDNA microarrays containing probes for 4503 rat genes. However, several hundred genes had to be discarded after data collection for various reasons. Each set of four microarrays corresponding to each rat was normalized and then summarized by using a robust summary statistic (methodology outlined later). The resultant array data will be referred to henceforth as an experiment. The dataset consists of log-expression levels on 3483 genes for 236 experiments (both ip and po) comprising 61 distinct compounds belonging to 8 hepatotoxicity classes, and 3 types of controls.

The data for this study can be decomposed in several ways. In this article we present the analysis for a subset of experiments in which the rats were dosed intraperitoneally. This dataset consisted of 3483 genes for 92 experiments on 2 controls (vehicle and untreated) and 25 compounds belonging to 5 hepatotoxicity classes: carcinogens $(\mathrm{CA})$, cholestasis $(\mathrm{CH})$, necrosis $(\mathrm{N})$, venocclusion $(\mathrm{V})$, and steatosis (S) inducers. Vehicle controls are labeled (A). Several compounds exhibit properties characteristic of more than one class, resulting in "fuzzy" classes. These compounds can legitimately be said to be belong to either class, although they may behave more strongly like one class. Because it was not possible to quantify the uncertainty, these compounds were taken to belong to the "dominant" class for purposes of training the classifier.

\section{DATA PREPROCESSING}

Microarray data need to be preprocessed to correct for various effects and biases detailed earlier (Amaratunga and Cabrera, 2004). Below we describe the data preprocessing that was performed in a sequence of stages [see Fig. 2 for a schematic diagram of the analysis procedure, and see Amaratunga and Cabrera, 2004 for further details of the methods used for steps P2 to P5].

P1. Check array quality. Construct a Spearman's trace plot to identify arrays or groups of arrays, which are substantially different from the rest. This is done by calculating, for each array, the median (and maximum) Spearman correlation coefficient between the array and all other arrays. These (median or maximum) correlations are then plotted against the arrays, which can be aligned according to various covariates, such as hybridization date and print batch numbers. Arrays that are pathological will typically have low correlations across the board and, therefore, stand out in the plot. 
Analysis Flow

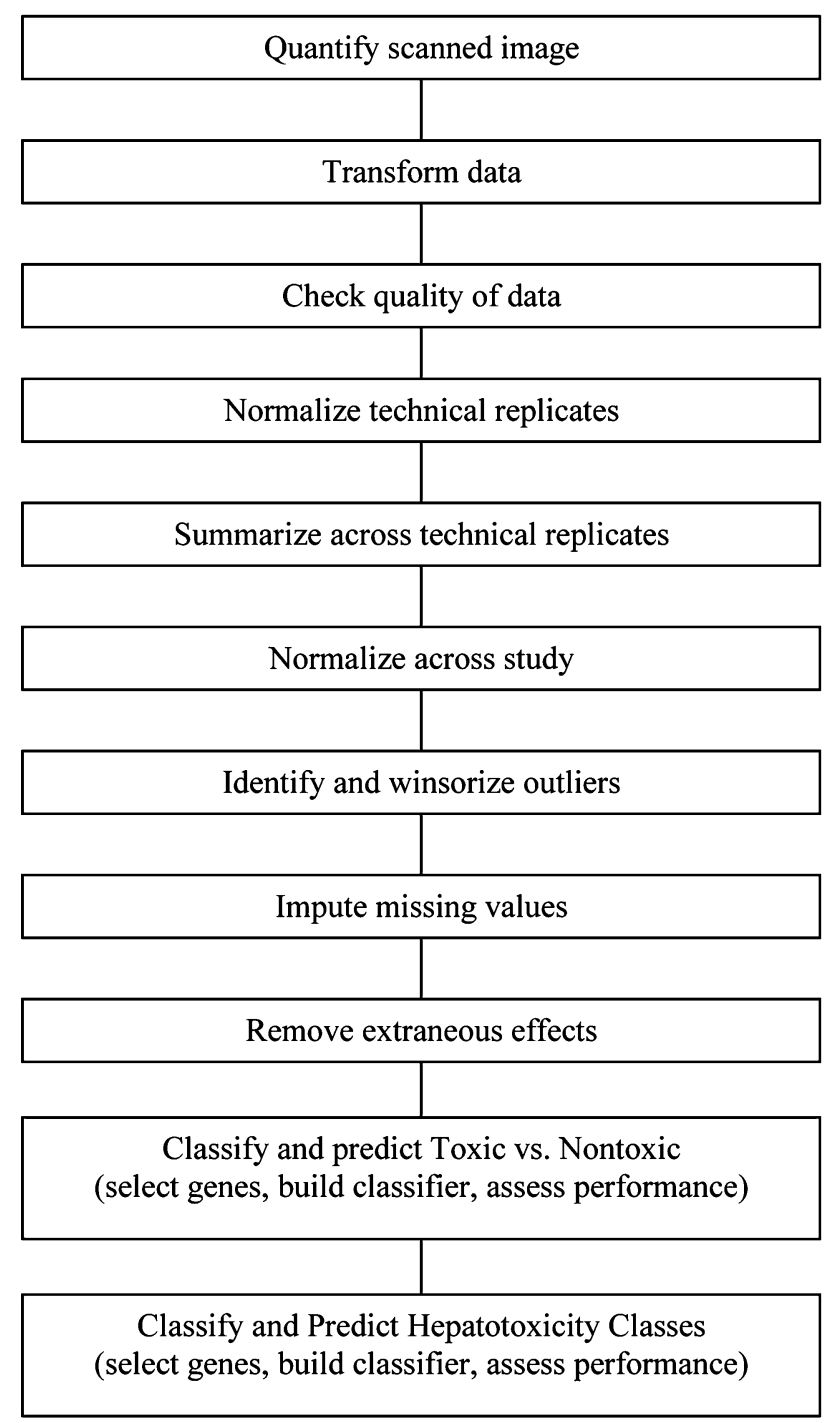

Figure 2 Flow-chart describing the steps in the data analysis.

P2. Transformation. Take logs to symmetrize the within-gene distribution. This transformation also reduces (but does not eliminate) the skewness of the acrossgene distribution but does not eliminate the heterogeneity of variances across genes.

P3. Normalize technical replicate. Performa lowess normalization across the technical replicates. Denoting an array by $X$, we first calculate a median "mock" array $M$ on $X_{i}, i=1, \ldots n$. For each array $X_{i}$, we fit a lowess smoother $g_{i}$ to the model $X_{i}=$ $\mathrm{g}_{i}(M)+\varepsilon_{i}$. The normalized array $X_{i}^{*}$ is given by: $X_{i}^{*}=f_{i}(M)$, where $g_{i}=f_{i}^{-1}$. 
P4. Summarize across technical replicate. We summarize the technical replicates by using a one-step biweight mean as described below. The biweight mean would be more resistant than the arithmetic mean to the outliers that are ubiquitous in microarray data and it has higher efficiency than the median under most distributions. It is calculated as follows. Let $X_{g i}$ denote the observation for gene $g$ on array $i$; and $M=\left\{M_{g}\right\}$, the median "mock" array. For each observation, we calculate $u_{g i}=\left(X_{g i}-M_{g}\right) / \tau S_{g}^{0}$, where $S_{g}^{0}$ is a smoothed resistant estimate of $\sigma_{g}$, the true gene variance, and $\tau$ is a tuning parameter. The biweight mean $B_{g}$ is then given by $B_{g}=\Sigma S_{g}^{0} w_{g i} X_{g i} / S_{g}^{0} w_{g i}$, where $w_{g i}=w\left(u_{g i}\right)$ is the biweight function.

P5. Normalize across study. Perform a quantile normalization across the entire study to align the distributions of all the arrays. This reduces monotonic nonlinear array effects across the arrays, but it is done in such a way that gene-specific effects are only slightly dampened. The normalization is done by first creating a median "mock" array $M$. For a given array $X_{i}$, the normalized array $X_{i}^{*}$ is obtained by binning each observation into percentile bins and backpredicting $X_{i}$ by linearly interpolating between the matched endpoints of the bins for $X_{i}$ and $M_{g}$.

P6. Identify and winsorize gross outliers among biological replicates. This reduces the impact of gross outliers on the subsequent classification. It is done as follows. Let $X_{g i}$ denote the observation for gene $g$ on array $i ; M_{g}=\operatorname{median}_{i}\left\{X_{g i}\right\}$; and $R_{g i}=X_{g i}-M_{g}$. Let $S_{g}^{\prime}$ be a smoothed resistant estimate of $\sigma_{g}$; the true gene variance obtained by a lowess prediction of $\left\{\left|R_{g i}\right|\right.$ vs. $\left.M_{g}\right\}$. Then, for a prespecified threshold $\tau$, the winsorized observations are obtained as:

$$
X_{g i}^{*}=\operatorname{median}\left(M_{g}-\tau S_{g}^{\prime}, X_{g i}, M_{g}+\tau S_{g}^{\prime}\right)
$$

P7. Impute values for missing data. Use a $k$ nearest neighbor procedure. For each gene with missing observations, first find its $k$ nearest neighbors based on Euclidean distances computed by using just those samples for which that gene is not missing and then impute the missing observations by averaging the corresponding nonmissing observations of its neighbors. Doing this imputation reduces the impact of missing values on downstream analysis.

P8. Remove extraneous effects such as the effect due to hybridization date. We do this by estimating using a median sweep. The residuals obtained by eliminating the effect(s) from the processed data above are then used as input for the classification procedure.

\section{CLASSIFICATION AND PREDICTION}

We now describe the classification methodology we used for this problem. As stated earlier, we used a two-stage strategy. In Stage I, we construct a classifier to classify a compound as toxic or nontoxic. Next, in Stage II, we construct a classifier to classify compounds designated as toxic into the various $(K)$ hepatotoxicity classes. We do this by using binary classifiers to classify each toxic compound into each of the $K(K-1) / 2$ two-way combination of classes. The votes of these classifiers are collated to make final class predictions for each test compound. For each binary classifier, the procedure consists of $\mathrm{C} 1$, a gene selection strategy to elicit 
the most relevant genes for differentiating between the two classes, and $\mathrm{C} 2$, linear discriminant analysis (LDA) to construct the classifier.

\section{Gene Selection}

Various strategies exist for gene selection. The strategy adopted and the number of genes selected tend to depend on the goal of the analysis. Often, finding the actual genes that are differentially expressed is of intrinsic interest in itself. This does not really place a constraint on the final number of genes selected. However, in this application, the goal is to use the differentially expressed genes to construct a classifier. Given the modest number of samples available per class, fairly aggressive filtering is required to avoid overfitting.

We use a two-pronged strategy to elicit genes for classification:

1. Eliminate genes whose variation relative to its mean is low. For each gene, we determine the mean and the range across classes. We then run a lowess smoother through the plot of gene ranges vs. means and eliminate any gene whose range does not exceed a prespecified percentage of the smoothed predicted range.

2. Eliminate genes that do not exhibit a significant between-class difference in a linear model fit with covariate and class (i.e., class vs. control) effects.

As a consequence of this twofold gene selection strategy, about 100 to 300 genes are retained per classification.

To validate that the selected genes were indeed meaningful in a hepatotoxicity context, we checked the gene ontologies of the genes selected for Stage I of the procedure. These genes of interest included several from pathways (lipid, sterol, and isoprenoid metabolism in particular) known to be disrupted by hepatotoxicants [see Table 1 and Hosack et al., 2003].

\section{Classification}

The data after preprocessing is classified using LDA. For binary classification, LDA seeks to find the linear projection of the data (in gene-space) that best separates the samples from the two classes using the following distance measure. By splitting the training part of the gene-expression matrix into the two classes, let $\bar{X}_{j}$ and $S_{j}$ denote the mean array and the array variance-covariance matrix for each class, $j=1,2$. Then the classification rule assigns array $X$ to Class 1 if $w^{\prime} X>w^{\prime}\left(\overline{X_{1}}+\overline{X_{2}}\right) / 2$; otherwise, array $X$ is assigned to Class 2. Here $w=S^{-}\left(\overline{X_{1}}+\right.$ $\left.\overline{X_{2}}\right)$ is the direction that maximizes the separation between the two classes. With microarray data, the number of genes selected will generally greatly exceed the number of available samples. Thus, the pooled variance-covariance matrix for the two classes, $S$, will be singular and a generalized inverse $S^{-}$of $S$ is used to compute $w$ [see Section 10.2 in Amaratunga and Cabrera, 2004 or Hand, 1997 for more details].

\section{Class Predictions}

Thus, our procedure constructs one binary classifier for Stage I, as well as 10 binary classifiers in Stage II, one for each of the two-way classifications for the five toxicity classes. 


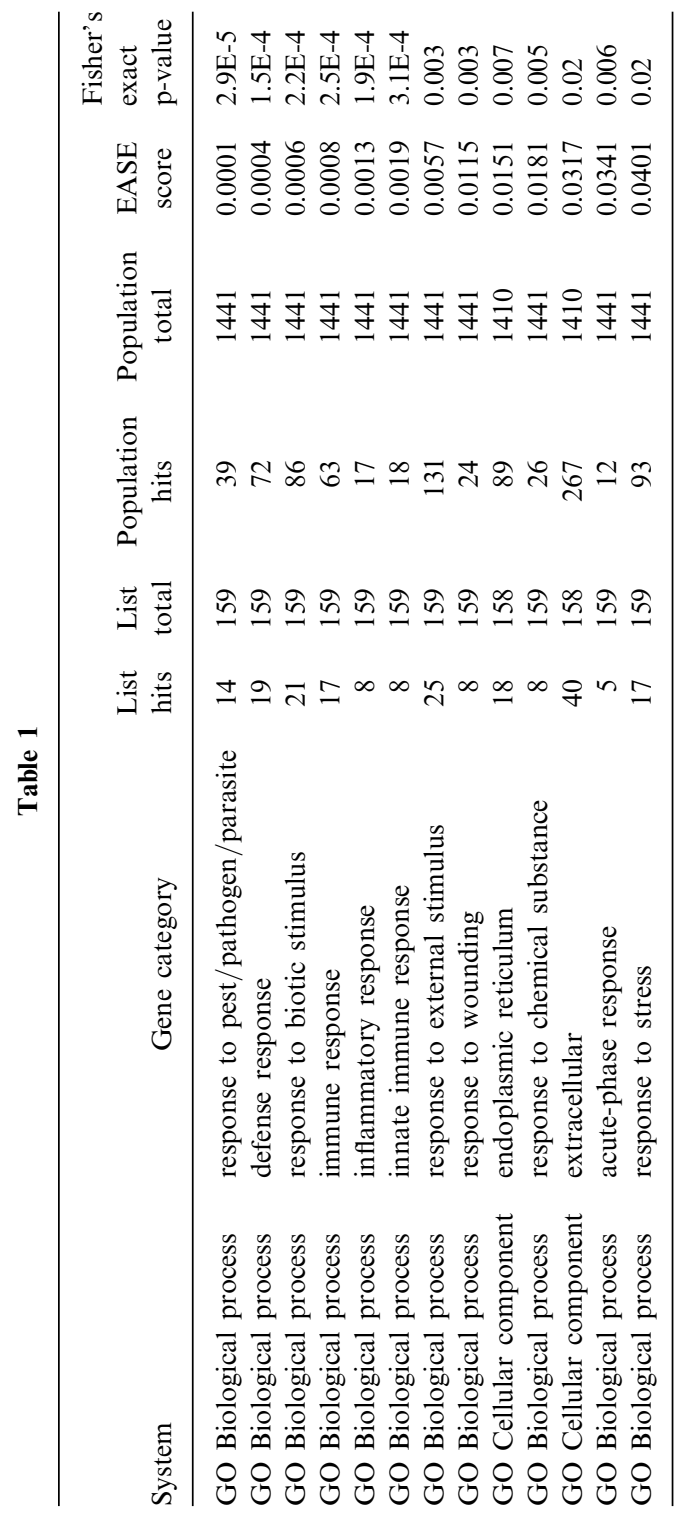


To predict the class memberships at Stage II, we collate the votes of the $K(K-1) / 2$ binary classifications. The test compound is assigned to the class with the maximum number of votes. In case of ties, the compound is assigned an undecided classification and not included in the calculation of error rates.

\section{Performance Assessment}

The apparent error rate (i.e., the proportion of samples misclassified by the classifier) at each stage is shown in Table 1. However, apparent error rates are well known to be overly optimistic. Therefore, we used cross-validation (CV) to get a better idea of the prediction error rate of the procedure (Hand, 1997) and indeed these values turned out to be higher than the apparent error rates.

We also assessed the effect of the gene selection on the apparent error rates by running the classifier as described above but without any gene selection.

We did two types of cross-validation. In leave-one-sample-out cross-validation (LOSO CV), each sample in turn was taken to be the test set and the rest of the samples were taken to form the training set; the results were collated to give an overall LOSO CV error rate. In leave-one-compound-out cross-validation (LOCO $\mathrm{CV}$ ), the set of all samples pertaining to each compound in turn was taken to be the test set, and the rest of the samples were taken to form the training set; the results were collated to give an overall LOCO CV error rate. Note that, because there were very few compounds in the classes $\mathrm{CH}, \mathrm{V}$, and $\mathrm{S}$, we were unable to include these classes in LOCO CV. Because the intent of the study was to assess the toxicity of a compound rather than that of a sample, LOCO CV is perhaps a more appropriate representation of the performance of the procedure than LOSO CV.

\section{RESULTS AND DISCUSSION}

Table 2 shows the overall apparent and CV error rates for (1) Toxic vs. Nontoxic in row 1, (2) the five hepatotoxicity classes in row 2, and (3) CA vs. N in row 3 (i.e., with classes $\mathrm{CH}, \mathrm{V}$, and $\mathrm{S}$ omitted). The columns correspond to (1) apparent, (2) LOSO CV, and (3) LOCO CV error rates. Table 2(a) refers to error rates for the classifiers run after preliminary gene selection and Table 2(b) refers to the same error rates for the classifiers run without any prior gene selection, thus providing a direct evaluation of the gene selection aspect of our procedure.

The plots in Figs. 3, 4, and 5 illustrate three-dimensional projections of the samples for various classifications. The projected dimensions correspond to a principal component decomposition of the covariate (gene) space for the subset of genes selected for LDA classification. The plots help understand the results in Table 2, because to some extent, one would expect that the degree of overlap among the classes, as reflected in the plots, would correspond to the degree of success of the classifiers, as reflected in the error rates.

Referring to Table 2(a), the apparent error rate for classifying Toxic vs. Nontoxic is $2.2 \%$, indicating that this procedure is indeed successful at identifying toxic compounds. In particular, none of the toxic compounds was misclassified as nontoxic. The plot in Fig. 3 (Toxic vs. Nontoxic) suggests that although a few samples of each class may be firmly entrenched in the other class, the classes seem generally separable otherwise. This may help explain the $12 \%$ LOSO CV error rate. 
Table 2 Overall apparent and CV error rates with and without gene selection

\begin{tabular}{lccc}
\hline Error rates & Apparent error rate & LOSO CV & LOCO CV \\
\hline & (after gene selection) & & \\
Toxic vs. non-toxic & $2.2 \%$ & $12 \%$ & $6.7 \% *$ \\
Toxicity classes & $7.1 \%$ & $34.3 \%$ & $\mathrm{NA}$ \\
"CA" vs. "N" & $5.1 \%$ & $27.1 \%$ & $46.8 \%$ \\
& $($ all genes) & & \\
& $5.4 \%$ & $15.2 \%$ & $5.3 \%{ }^{* *}$ \\
Toxic vs. non-toxic & $14.7 \%$ & $38.2 \%$ & $\mathrm{NA}$ \\
Toxicity classes & $10.2 \%$ & $30.5 \%$ & $42.1 \%$ \\
"CA" vs. "N" & N,**: Note that this is computed only for "Non-Toxic" given "Toxic", since \\
LOCO-CV cannot be computed for the converse "Toxic" given "Non-toxic", \\
there being primarily one control compound.
\end{tabular}

The low LOCO CV error rate $(6.7 \%)$ indicates that even though some samples associated with a compound may be misclassified, the compound itself may not be, thereby underscoring the importance of replication. Note that LOCO CV is computed only for Nontoxic given Toxic, because LOCO CV cannot be computed for the converse (Toxic given Nontoxic), there being only one compound in the latter category. The low LOCO CV error rate is consistent with a $0 \%$ apparent error rate that was observed for Nontoxic given Toxic and is very heartening to note, especially from the standpoint of screening compounds for safety.

The $7.1 \%$ and $5.1 \%$ apparent error rates for the classes, and for CA vs. N, respectively, indicates that the classification procedure is also quite successful at identifying the different hepatotoxicity mechanisms, although the performance of this classification is evidently not quite as good as that of Toxic vs. Nontoxic. The

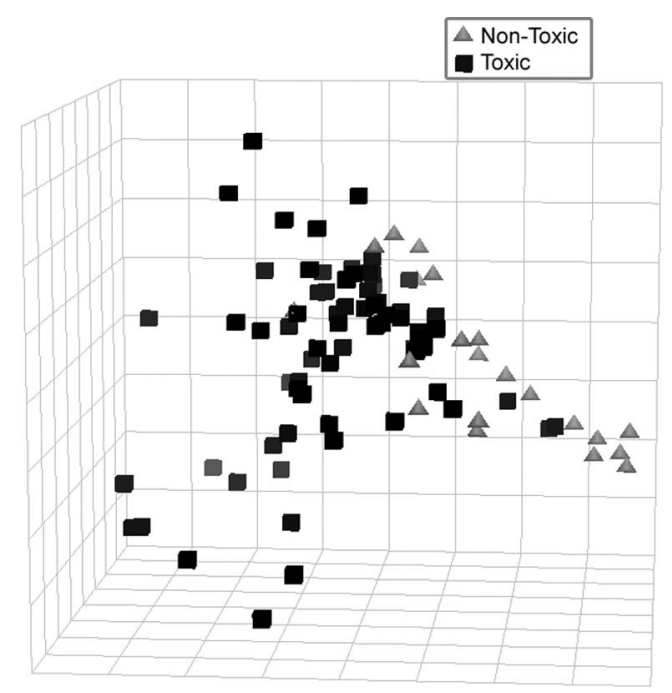

Figure 3 View of samples corresponding to toxic and non-toxic classes in a 3-D principal components projection. 


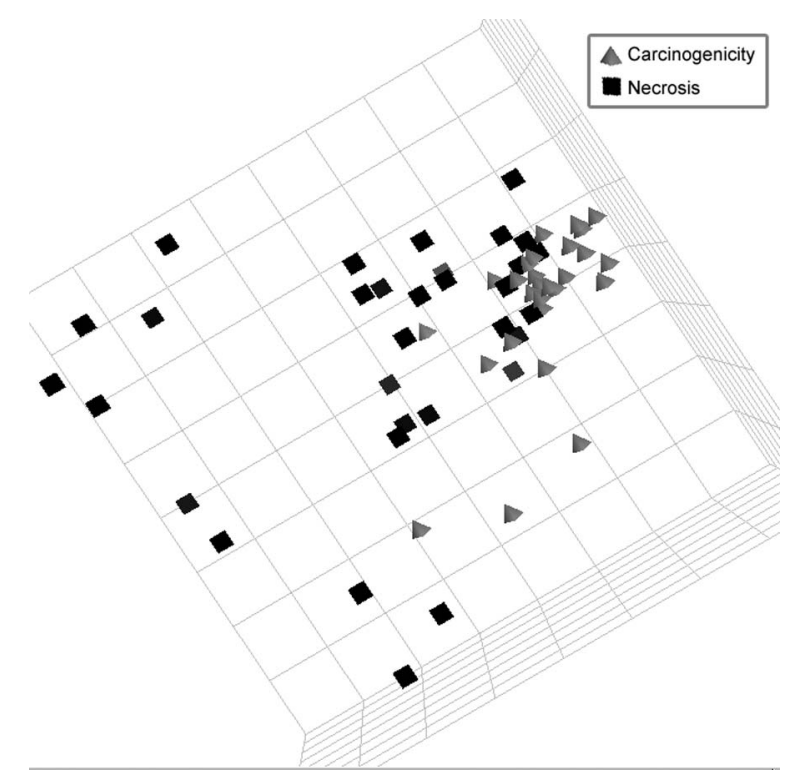

Figure 4 View of samples corresponding to "carcinogen" and "necrosis" classes in a 3-D principal components projection.

two plots in Figs. 4 and 5 may help explain why. They indicate substantially greater overlap among the classes, with a significant portion of the overlap appearing to be between the highly represented, and possibly diverse, classes CA and N (Fig. 5). In addition, class $\mathrm{S}$ appears to be embedded within these two classes. This observation is also consistent with the much higher LOSO CV error rates $(34.3 \%$ and $27.1 \%$, respectively). Although emphasizing that the plots just represent a three-dimensional projection of a much higher dimensional space used for LDA classification, they do suggest that this is a challenging dataset to classify.

Overall, gene selection enhances the performance of the classifiers. Apparent error rates were reduced by close to $50 \%$ after gene selection $(60 \%, 52 \%$, and $45 \%$ reduction, respectively, for Toxic vs. Nontoxic, the hepatotoxicity classes and CA vs. $\mathrm{N})$. Improvements in the LOSO CV error rates were more modest $(21 \%, 10 \%$, and $11 \%$, respectively for Toxic vs. Nontoxic, the hepatotoxicity classes and CA vs. N) after gene selection. The LOCO CV error rate seemed to increase slightly after gene selection. Although one should keep in mind the inherent variability in $\mathrm{CV}$ when interpreting these results, the results do seem to suggest that perhaps gene selection becomes more important and needs to be more nuanced, as the complexity of the classification increases.

Although the general reduction in $\mathrm{CV}$ error rates as a result of gene selection is gratifying, it is probably more heartening, and of more immediate interest to the scientists, to note that the selected genes appear to be concentrated in pathways known to be disrupted by hepatotoxicants. This is illustrated in Table 1. Columns 1 and 2 in this table refer to which gene ontology comes into play and which system in particular is affected. Columns 3 and 4 refer to the number of genes in 


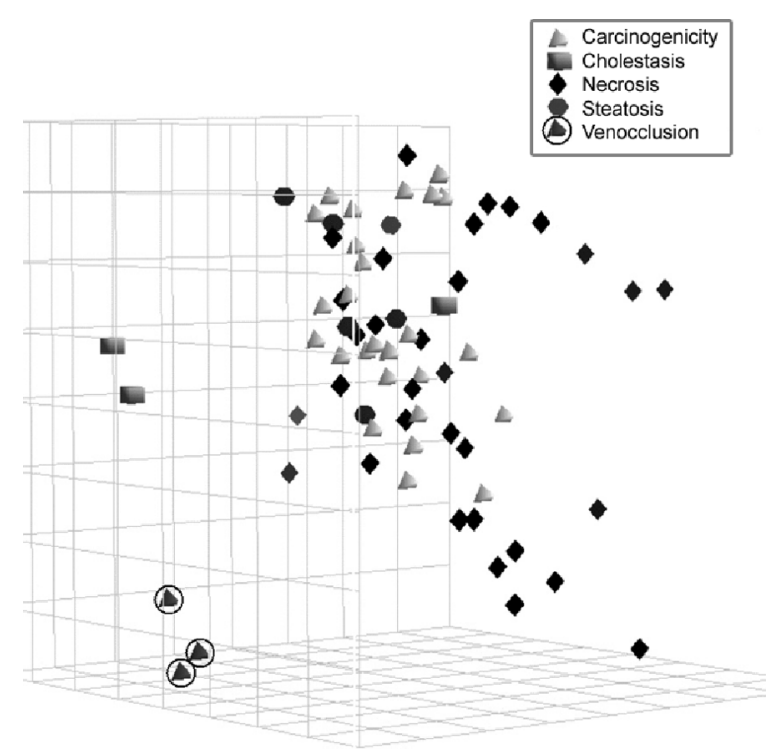

Figure 5 View of samples corresponding to five hepatotoxicity classes in a 3-D principal components projection.

the selected gene list that are in the systems corresponding to columns 2 and 1 , respectively. Similarly, columns 5 and 6 refer to the number of genes in the entire gene list that are in the systems corresponding to columns 2 and 1, respectively. Columns 7 and 8 give the EASE score (10) and the $p$-value for the Fisher exact test, respectively. The EASE score is the $p$-value associated with a variant of the onetailed Fisher exact probability for measuring overrepresentation of genes associated with the specific process in the gene list, relative to their representation in the gene list not associated with the specific process. Both the Fisher $p$-value and the EASE score can be viewed as indicators of the success of the gene selection strategy in eliciting genes in relevant pathways. The rows of the table were thresholded at an EASE score of 0.05. As an illustration, let us consider the first row in Table 1. Of the 3483 genes in the entire list, 1441 (column 6) were annotated for biological processes, and of these, 39 (column 5) were associated with response to pest/pathogen/parasite. Correspondingly, of 341 genes in the selected gene list, 159 (column 4) were annotated for biological processes, and of these 14 (column 3) were associated with response to pest/pathogen/parasite. The EASE score is then calculated for the corresponding $2 \times 2$ table, with entries 14 and 145 in row 1 for genes associated with response to pest/pathogen/parasite, and entries 25 and 1257 in row 2 for genes not associated with response to pest/pathogen/parasite, in the selected gene list and not-in-selected gene list categories respectively.

The highly significant EASE and Fisher $p$-value scores in columns 7 and 8 strongly attest to the biological relevance of our gene selection strategy. The genes that were selected for distinguishing classes of hepatotoxicants from nontoxicants are heavily weighted toward genes that are involved in responses to environmental challenges (Table 1), rather than toward those genes that are constitutively active 
and important for maintaining normal, quiescent metabolic processes. This is not surprising because animals have evolved distinct coordinated gene responses to varying insults, and this repertoire of genes is induced or repressed to different degrees depending on the transcription factors that are activated or inhibited by various compounds and their metabolites.

The premise underlying the study was that, at very high doses of toxicants, gene expression changes at 24 hours presage many histopathological endpoints observed later and could, therefore, be used to identify hepatotoxicity and, if hepatotoxic, the mechanism underlying hepatotoxicity. The results above indicate that such a procedure can be used to this end to identify general hepatotoxicity quite successfully and to classify specific mechanisms of hepatotoxicity to some extent. As technology for functional genomics investigations evolves, it is to be expected that toxicogenomics will introduce a new dimension to the study of toxicity.

\section{REFERENCES}

Amaratunga, D., Cabrera, J. (2004). Exploration and Analysis of DNA Microarray and Protein Array Data. New York: John Wiley.

Dudoit, S., Fridlyand, J., Speed, T. P. (2002). Comparison of discrimination methods for the classification of tumors using gene expression data. Journal of the American Statistical Association. 97(457):77-87.

Friedman, J. (1996). Another Approach to Polychotomous Classification. Technical Report. Stanford University.

Hand, D. J. (1997). Construction and Assignment of Classification Rules. Chichester: John Wiley.

Hastie, T., Tibshirani, R., Friedman, J. (2001). Elements of Statistical Learning. New York: Springer Verlag.

Hosack, D. A., Dennis, G., Jr., Sherman, B. T., Lane, H. C., Richard, R. A. (2003). Identifying biological themes within lists of genes with EASE. Genome Biology 4(9):R60.

McMillian M., Nie, A. Y., Parker, J. B., Leone, A., Kemmerer, M., Bryant, S., Herlich, J., Yieh, L., Bittner, A., Liu, X., Wan, J., Johnson, M. D. (2004a). Inverse gene expression patterns for macrophage activating hepatotoxicants and peroxisome proliferators in rat liver. Biochemical Pharmacology 67(11):2141-2165.

McMillian, M., Nie, A. Y., Parker, J. B., Leone, A., Kemmerer, M., Bryant, S., Herlich, J., Liu, Y., Yieh, L., Bittner, A., Liu, X., Wan, J., Johnson, M. D. (2004b). A gene expression signature for oxidant stress/reactive metabolites in rat liver. Biochemical Pharmacology 68:2249-2261.

Nuwaysir, E. F., Bittner, M., Trent, J., Barrett, J. C., Afshari, C. A. (1999). Microarrays and toxicology: the advent of toxicogenomics. Molecular Carcinogenesis 24:153-159.

Speed, T. (2003). Statistical Analysis of Gene Expression Microarray Data. New York: Chapman and Hall. 